\title{
SURVIVAL TIME AND HISTOLOGICAL OUTCOME OF MAJOR ORGANS FOLLOWING HONEY ADMINISTRATION TO ACUTE PARAQUAT-INTOXICATED RATS: A PRELIMINARY STUDY
}

\author{
SUK PENG TANG ${ }^{a}{ }^{*}$, HASNAN JAAFAR ${ }^{\mathrm{b}}$, SIEW HUA GANc, KUTTULEBBAI N. S. SIRAJUDEENd, \\ SITI AMRAH SULAIMAN ${ }^{\mathrm{a}}$
}

aDepartment of Pharmacology, bDepartment of Pathology, cHuman Genome Center, dDepartment of Chemical Pathology, School of Medical Sciences, Health Campus, Universiti Sains Malaysia, 16150 Kubang Kerian, Kelantan, Malaysia

Email: sp_t84@yahoo.com

Received: 30 Sep 2016 Revised and Accepted: 05Jan 2017

\section{ABSTRACT}

Objective: The objective of this research was to investigate the possible protective effect of Tualang honey (TH) in acute paraquat (PQ) toxicity in rats.

Methods: A total of 48 male Sprague-Dawley rats aged eight weeks old were used. Oral PQ and TH were administered at $225 \mathrm{mg} / \mathrm{kg}$ and $0.2 \mathrm{~g} / \mathrm{kg}$, respectively. The effects of single and multiple TH treatmentson PQ-intoxicated rats were then investigated. Single TH treatment groups received $\mathrm{TH}$ at $0.5(\mathrm{PQ}+\mathrm{TH} 0.5 \mathrm{~h}), 2(\mathrm{PQ}+\mathrm{TH} 2 \mathrm{~h})$ or $6(\mathrm{PQ}+\mathrm{TH} 6 \mathrm{~h})$ hours following PQ administration. Multiple TH treatment groups received TH at $0.5,2$ and 6 $\mathrm{h}(\mathrm{PQ}+\mathrm{THtrp})$ or further daily treatment for the following six days (PQ+TH7d) after $\mathrm{PQ}$ administration ( $\mathrm{n}=6$ per group). The survival time of the rat was recorded until day 28 before sacrifice, which was followed by a histological examination.

Results: Treatment with TH did not improve the survival rate of PQ-intoxicated rats. However, the median survival time of rats that received multiple TH treatments was significantly longer compared to that of the PQ+TH6h group. TH treatment was found to improve the histological outcomes of PQ-intoxicated rats, particularly in the lungs.

Conclusion: Our findings suggest the potential role of honey in delaying the toxic effects of PQ.

Keywords: Paraquat, Acute toxicity, Tualang honey

(C) 2016 The Authors. Published by Innovare Academic Sciences Pvt Ltd. This is an open access article under the CC BY license (http://creativecommons.org/licenses/by/4. 0/) DOI: http://dx.doi.org/10.22159/ijpps.2017v9i2.15466

Although paraquat (PQ) is a widely used herbicide, the challenges resulting from PQ exposure are reported all over the world and are mainly caused by suicidal intent, accidental poisoning or occupational exposures [1]. PQ is known to stimulate the production of various reactive oxygen species (ROS) via a single electron redox cycle in vivo[2, 3]. ROS readily attack key cellular structures and molecules, thus causing cellular deleterious effects that form the basis of various disease conditions $[4,5]$.

To date, there is no specific antidote clinically available for $P Q$ poisoning. The conventional approach in treating $\mathrm{PQ}$ poisoning focuses on three main areas, which include the prevention of absorption from the gastrointestinal tract, the enhancement of elimination of PQ from the body and the administration of therapies directed against toxicity. Nevertheless, these treatment methods have been disappointing, with very high mortality rates [1, 6-9]. Many studies have been conducted in an effort to search for an effective antidote for $P Q$ poisoning with several being directed towards the use of antioxidants because PQ induces its toxic effect via oxidative stress-mediated mechanisms [10-14].

Honey, which is one of the oldest foods consumed over the centuries with potential therapeutic properties because of its high antioxidant capacity, has gained substantial research interest in the past decade [15]. Honey contains both aqueous and lipophilic antioxidants, and interaction between these antioxidants suggests honey's potential as an ideal natural antioxidant that can act at various cellular sites in the case of $P Q$ poisoning $[16,17]$. Tualang honey (TH) is a wild honey harvested from Tualang trees found in many Asian rainforests. Various studies have been conducted in an effort to evaluate the possible medicinal uses of TH [18-24]. One of the proposed therapeutic values is attributed partly due to its antioxidant properties. A study by Mohamedet al.[25] showed that $\mathrm{TH}$ contains phenolic compounds with strong antioxidant activities. However, to date, the protective effects of honey on PQ toxicity have not been investigated. Therefore, the aim of this study is to investigate the potential beneficial effects of TH in acute PQ poisoning in an animal model.

TH (AgroMas $囚$ ) used in this study was supplied by the Federal Agricultural Marketing Authority (FAMA), Kedah, Malaysia. The honey samples used in this study were filtered, concentrated to 20 $\%(\mathrm{w} / \mathrm{v})$ water content at $40{ }^{\circ} \mathrm{C}$ and sterilized by gamma irradiation at $25 \mathrm{kGy}$ [Sterilgamma (M) Sdn. Bhd., Selangor, Malaysia]. The same batches of honey were used throughout the analysis. The chemical characteristics including the antioxidant properties of the investigated honey have been previously described [25].

Male Sprague-Dawley rats $(n=48)$ aged eight weeks old were purchased from the Animal Research and Service Center, Health Campus, USM, Kubang Kerian, Kelantan, Malaysia. All animals were individually housed in polypropylene cages in a well-ventilated room maintained at a $12 \mathrm{~h}$ light/dark cycle and $25 \pm 2{ }^{\circ} \mathrm{C}$ room temperature. Food pellets and water were given ad libitum unless otherwise stated. All rats were acclimatized to the room condition for at least $1 \mathrm{w}$ prior to the experiment. This study was approved by the Animal Ethics Committee, Universiti Sains Malaysia [Approval No.: USM/Animal Ethics Approval/2009/(45)(140)], which is in accordance with the Institutional Guideline for the Care and Use of Animals for Scientific Purposes.

The rats were randomly divided into eight groups of six rats each. Groups A and B were administered $0.5 \mathrm{ml}$ of double deionized water $\left(\mathrm{ddH}_{2} \mathrm{O}\right)$, whereas Groups $\mathrm{C}, \mathrm{D}, \mathrm{E}, \mathrm{F}, \mathrm{G}$ and $\mathrm{H}$ were administered PQ (Sigma-Aldrich, St Louis, MO, USA; $225 \mathrm{mg} / \mathrm{kg}$, p. o.) at the beginning of the study $(\mathrm{t}=0)$. The rats were then treated with either $\mathrm{ddH}_{2} \mathrm{O}$ or TH $(0.2 \mathrm{~g} / \mathrm{kg}$, p. o. $)$ at the specific time intervals described in table 1 . The doses of $P Q$ and TH were determined based on preliminary studies conducted in our laboratory (data not shown). 
Table 1:Rat grouping and type of treatment in all experimental groups

\begin{tabular}{ll}
\hline Group & Treatment received after PQ administration* \\
\hline $\mathrm{A}(\mathrm{N})$ & $\mathrm{ddH}{ }_{2} \mathrm{O}$ at $2 \mathrm{~h}$ \\
$\mathrm{~B}(\mathrm{TH})$ & $\mathrm{TH}$ at $2 \mathrm{~h}$ \\
$\mathrm{C}(\mathrm{PQ})$ & $\mathrm{ddH}_{2} \mathrm{O}$ at $2 \mathrm{~h}$ \\
$\mathrm{D}(\mathrm{PQ}+\mathrm{TH} 0.5 \mathrm{~h})$ & $\mathrm{TH}$ at $0.5 \mathrm{~h}$ \\
$\mathrm{E}(\mathrm{PQ}+\mathrm{TH} 2 \mathrm{~h})$ & $\mathrm{TH}$ at $2 \mathrm{~h}$ \\
$\mathrm{~F}(\mathrm{PQ}+\mathrm{TH} 6 \mathrm{~h})$ & $\mathrm{TH}$ at $6 \mathrm{~h}$ \\
$\mathrm{G}(\mathrm{PQ}+\mathrm{THtrp})$ & $\mathrm{TH}$ at $0.5,2$ and $6 \mathrm{~h}$ \\
$\mathrm{H}(\mathrm{PQ}+\mathrm{TH} 7 \mathrm{~d})$ & TH at $0.5,2$ and $6 \mathrm{~h}$ and once daily for the \\
\hline
\end{tabular}

*Groups A and B (non-PQ treated) were administered $\mathrm{ddH}_{2} \mathrm{O}(0.5 \mathrm{ml}$, p. o.), whereas groups C, D, E, F, G and H (PQ-treated) were administered PQ (225 mg/kg, p. o.) at $\mathrm{t}=0$. The dose of TH used was 0.2 $\mathrm{g} / \mathrm{kg}, \mathrm{n}=6$ per group. [Abbreviations: $\mathrm{ddH}_{2} \mathrm{O}$, double distilled water; $\mathrm{PQ}$, paraquat; $\mathrm{TH}$, Tualang honey]

All rats were closely observed for a maximum period of $28 \mathrm{~d}$ before being sacrificed. The survival time after PQ administration of each rat was recorded until day 28. All moribund animals or animals showing severe signs of toxicity were sacrificed. All surviving animals were sacrificed by decapitation under deep anaesthesia with ketamine $(90 \mathrm{mg} / \mathrm{kg})$ and xylazine $(5 \mathrm{mg} / \mathrm{kg})$ at the end of the experimental period. The lungs, kidneys and liver of all rats (moribund and surviving) were collected and fixed in $10 \%$ formalin solution (Merck, Germany). They were then processed for subsequent histopathological analysis by hematoxylin and eosin $(\mathrm{H}$ and E) staining.

Kaplan-Meier survival analysis with Log-Rank pairwise comparison test was performed using the IBM Statistical Package for the Social Sciences (SPSS) software version 22.0 (Armonk, NY, USA) to evaluate the median differences between the experimental groups.

This study is the first to report on the effect of honey administration on PQ intoxication in rats. Table 2 shows the effects of administering various honey dosing regimens on the median survival time of PQintoxicated rats. Overall, TH treatment did not improve the survival rate of $\mathrm{PQ}$-intoxicated rats, regardless of the time of administration, with mortality rates remaining high $(>60 \%)$. However, the rats that received triple honey treatments at $0.5,2$ and $6 \mathrm{~h}$ (group PQ+THtrp) or continuous daily honey treatment for seven days (group PQ+TH7d) following PQ administration had a significantly longer median survival time compared to animals that received only a single honey treatment at six hours following PQ intoxication (group $\mathrm{PQ}+\mathrm{TH} 6 \mathrm{~h}$ ). In comparing the groups that received only single honey treatments (at $30 \mathrm{~min}, 2 \mathrm{~h}$ or $6 \mathrm{~h}$ ), there was a trend in which the earlier the animals were administered the honey treatment, the longer the observed median survival time.

Table 2: The effects of administering various honey dosing regimens on the median survival time of PQ-intoxicated rats

\begin{tabular}{lll}
\hline Group & Survival (\%) & Median (95\% CI) (days) \\
\hline PQ & 12.5 & $3.50(2.53,4.47)$ \\
PQ+TH0.5h & 16.7 & $4.00(1.84,6.16)$ \\
PQ+TH2h & 33.3 & $3.10(1.18,5.02)$ \\
PQ+TH6h & 0.0 & $2.50(1.78,3.22)$ \\
PQ+THtrp & 16.7 & $4.50(2.10,6.90)^{* *}$ \\
PQ+TH7d & 33.3 & $5.30(2.90,7.70)^{*}$ \\
\hline
\end{tabular}

${ }^{*} p<0.05 ;{ }^{* *} p<0.01$ compared to group PQ+TH6h (Log Rank pairwise comparison)

The death associated with multiple organ failures as observed in most cases of severe PQ poisoning was evidenced by post-mortem histological analyses of the lung, kidney and liver of rats, particularly in the rats that died within four \&ays $86(\mathrm{~h})$ following PQ intoxication, with or without honey treatments. Features of circulatory disturbances including congestion, haemorrhage and edema were observed in all three major organs. The representative photomicrographs of $\mathrm{H}$ and $\mathrm{E}$ stained lung sections from all the experimental groups are shown in fig. 1(A-C). Microscopic examination of the rats' lungs indicated marked alveolar collapse, vascular congestion and severe pulmonary edema in rats that were found to be dead or were sacrificed within four days $6(\mathrm{~h})$ following $P Q$ intoxication due to severe distress symptoms. However, rats that received three honey treatments (PQ+THtrp) showed relatively normal structure but a higher number of red blood cells and mild congestion [fig. 1(A)]. In rats found dead or sacrificed within four to seven days (96-168 h) after PQ intoxication, thicker alveolar septa with inflammatory cell infiltrations and interstitial edema were observed in the PQ group. In comparison to the PQ group, the lung of rats that received honey treatments showed relatively normal alveolar structures with major histological changes that include infiltration of inflammatory cells in the alveolar space and mild congestion [fig. 1(B)]. The histological findings of the lungs generally corresponded to the increased respiratory rate and abdominal breathing as observed in rats that were exposed to PQ and were found dead or sacrificed earlier. In the rats sacrificed at the end of the experimental period (28 d after PQ-intoxication), rats from the PQ group showed a distorted lung structure with collapsed or loss of alveolar space, in combination with some compensatory expanded alveolar space. Additionally, a thickening of the alveolar walls with inflammatory cell infiltration, usually an initial event in pulmonary fibrosis, was also observed. These pathological changes were also observed in PQ-intoxicated rats that received honey treatments but to a lesser extent [fig. 1(C)]. This finding suggests that honey may be involved in delaying the progression of lung fibrosis, one of the most common fatal complications in PQ poisoning. Although there is a lack of effective treatments to prevent or reverse the progression of pulmonary fibrosis [26, 27], it will be of research interest to investigate the possible protective effects of honey against PQ-induced pulmonary fibrosis.

The representative photomicrographs of the $\mathrm{H}$ and $\mathrm{E}$ stained kidney sections from all the experimental groups are shown in fig. 2(A-C). Microscopic examination of the kidney of rats found dead or sacrificed earlier within four days $(\leq 96 \mathrm{~h})$ following PQ intoxication revealed features of acute tubular necrosis, although the architecture of a glomerulus and the surrounding tubules was still recognisable despite the dissolution of the tubule nuclear material. Mild haemorrhage and congestion were also observed [fig. 2(A)]. In the rats found dead or sacrificed earlier within four to seven days (96-168 h) following PQ intoxication, features of acute tubular necrosis and mild congestion were observed in the PQ-intoxicated rats with or without honey treatment but to a lesser extent compared to rats found dead or sacrificed within four days [fig. 2(B)]. In the rats sacrificed at the end of the experimental period (28 $\mathrm{d}$ following PQ intoxication), the PQ group showed a normal tubular structure with mild tubular necrotic changes. PQ-intoxicated rats that received honey treatment showed minimal histopathological changes and were comparable to those of the healthy control groups [fig. 2(C)]. Additionally, increased urinary excretion (wet bedding) was observed in some, but not all, of the rats that received TH treatment for a few days following PQ intoxication. The rats tended to survive until the end of $28 \mathrm{~d}$, suggesting that some recovery from acute renal injuries might have occurred following honey treatment.

The representative photomicrographs of the liver sections from all the experimental groups are shown in fig. $3(\mathrm{~A}-\mathrm{C})$. The major histological changes observed in the liver of rats found dead or sacrificed earlier within four days ( $\leq 96 \mathrm{~h}$ ) following PQ intoxication include dilated and 
congested liver sinusoids [fig. 3(A)]. Additionally, there was an increase in the number of binuclear hepatocytes in all PQ-intoxicated groups, but the increment is comparatively smaller in groups that received multiple honey treatments. Similar histological changes were observed in the liver of rats found dead or sacrificed earlier within four to seven days (96-168 h) following PQ intoxication [fig. 3(B)]. In the rats that survived until the end of the experimental period $(28 \mathrm{~d}$ following PQ-intoxication), no evidence of major histological changes was observed among the experimental groups [fig. 3(C)].Liver injury may promote regenerative activity, which is relatively rare in healthy adult hepatocytes [28]. The increase in the major dividing cell type during liver regeneration may be a result of the cellular response to PQ-induced hepatotoxicity. PQ-induced karyomegaly and binucleation in the mouse liver was previously shown in caspase- 2 knockout mice that had a reduced ability to withstand oxidative stress and were more susceptible to various oxidative stimuli [29].

Altogether, these findings suggest that treatment with TH may reduce the toxic actions of PQ. Further investigation of the oxidative stress parameters such as the enzyme activities of superoxide dismutase, catalase, and glutathione peroxidase, as well as malondialdehyde content, should be conducted to elucidate the exact mechanisms. Nevertheless, the individual rats responded differently to $P Q$, with the earliest fatalities recorded at $10 \mathrm{~h}$ post PQ intoxication, followed by within days and $1 \mathrm{w}$, with some animals even surviving until the end of the experimental period. The inter-individual differences in responses made acomparison between the rats a challenge. Additionally, the histological findings based on early death $(\leq 96 \mathrm{~h})$, delayed death (up to $1 \mathrm{w}$ ) and survival for the experimental period represented a small number of rats in each experimental group. Therefore, the relatively smaller sample size in this study may not reflect the actual response to PQ or TH. Moreover, although TH seemed to prolong the rats' survival time to a certain extent and improved the histological outcomes compared to animals that received PQ only, the mortality rate remained high. Therefore, a new therapeutic approach is still needed in which the use of honey can be incorporated with other therapeutic agents (i.e., honey as an adjunctive treatment).

(A)

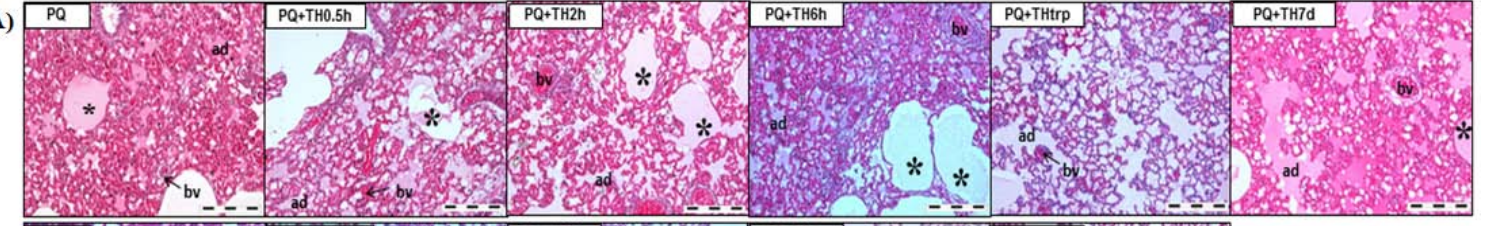

(B)

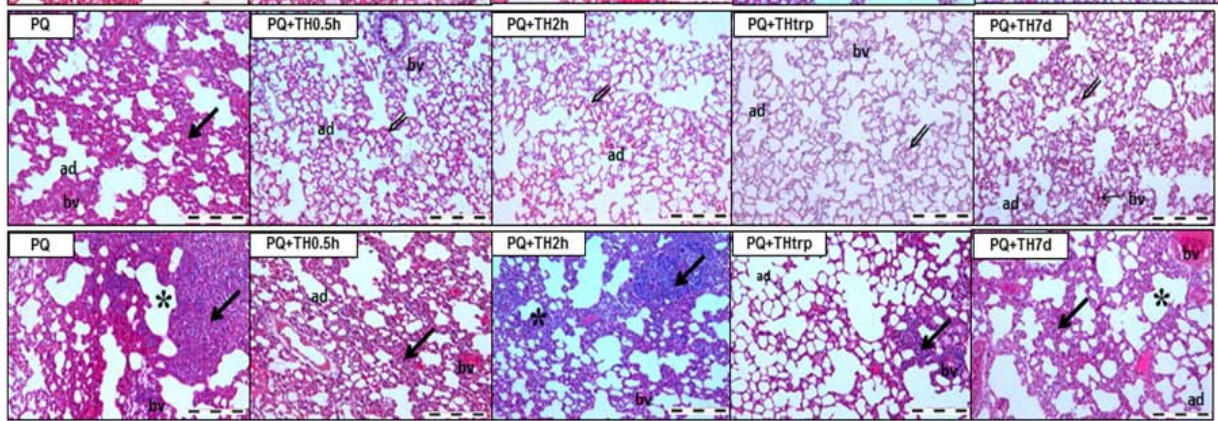

Fig. 1: Representative photomicrographs of $\mathrm{H}$ and $\mathrm{E}$ stained lung section from rats sacrificed or found dead (A) within four days ( $\leq 96 \mathrm{~h})$, (B)within four to seven days (96-168 h) and (C) at the end of the experimental period (day 28) following PQ intoxication (scale bar: 200 $\mu \mathrm{m}$; original magnification: $\times \mathbf{1 0 0}$ ). The major histopathological changes include distorted alveolar configuration or large distended air spaces $(*)$, vascular congestion and pulmonary edema (alveolar filled with pink/pale-eosinophilic transudate), diffuse alveolar wall thickening (thick arrow) with interstitial inflammation and edema, infiltration of inflammatory cells in the alveolar space and mild congestion (double arrow). [ad, alveolar duct; bv, blood vessel; PQ, paraquat group; TH, Tualang honey (single-dose honey treatment at 0.5 (TH0.5h), 2 (TH2h) or 6 h (TH6h); multiple-dose honey treatment at $0.5,2$ and 6 h (THtrp) or $7 \mathrm{~d}$ of consecutive treatment (TH7d)]

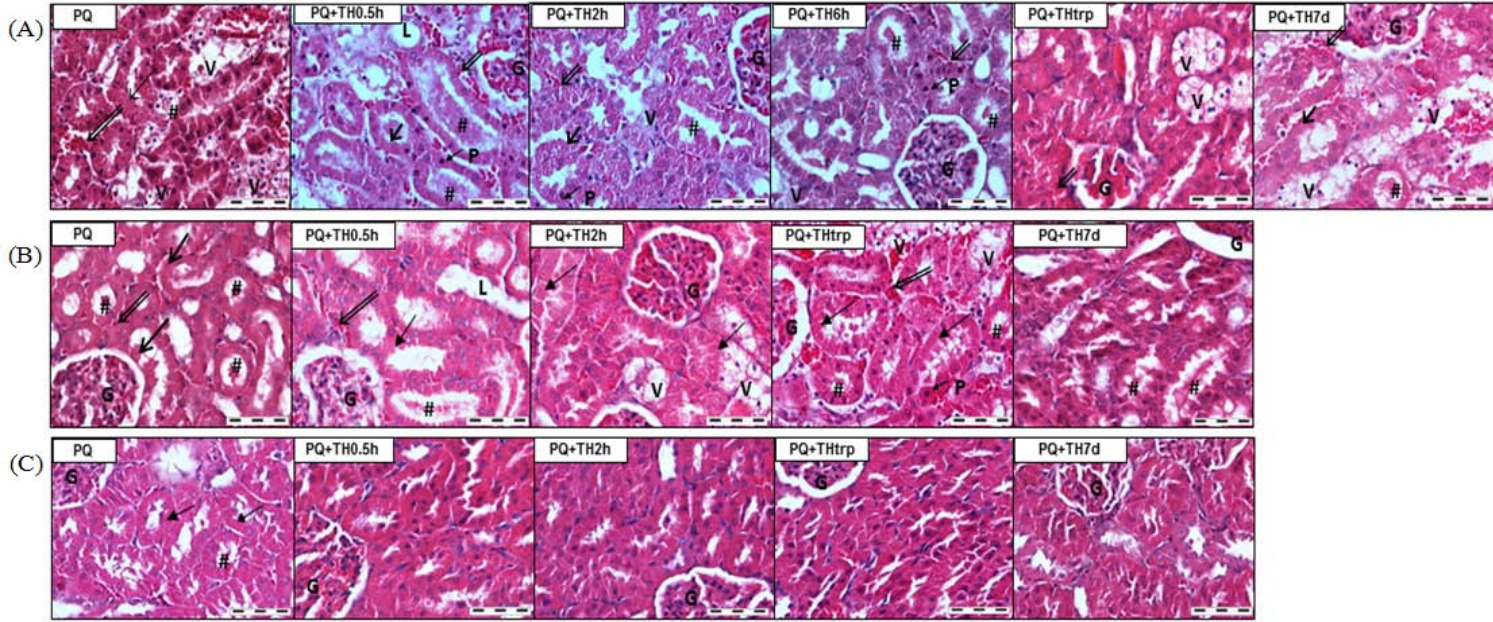

Fig.2: Representative photomicrographs of $\mathrm{H}$ and $\mathrm{E}$ stained kidney section from rats sacrificed or found dead (A) within four days ( $\leq 96 \mathrm{~h}$ ), $\mathrm{B}$ ) within four to seven days (96-168 h) and (C) at the end of the experimental period (day 28) following PQ intoxication (scale bar: $50 \mu \mathrm{m}$; original magnification: $\times 400$ ). The major histopathological changes include features of acute tubular necrosis [pyknotic nuclei (P), loss of nuclei (arrow),

or vacuolated cytoplasm $(\mathrm{V})$, tubular protein cast $(\#)$ and mild congestion (double arrow) were observed in the $\mathrm{PQ}$-intoxicated rats with or

without honey treatments. [G, glomerulus; L-tubular lumen; PQ, paraquat group; TH, Tualang honey (single-dose honey treatment at 0.5

(TH0.5h), 2 (TH2h) or 6 h (TH6h); multiple-dose honey treatment at 0.5, 2 and 6 h (THtrp) or $7 \mathrm{~d}$ of consecutive treatment (TH7d)] 


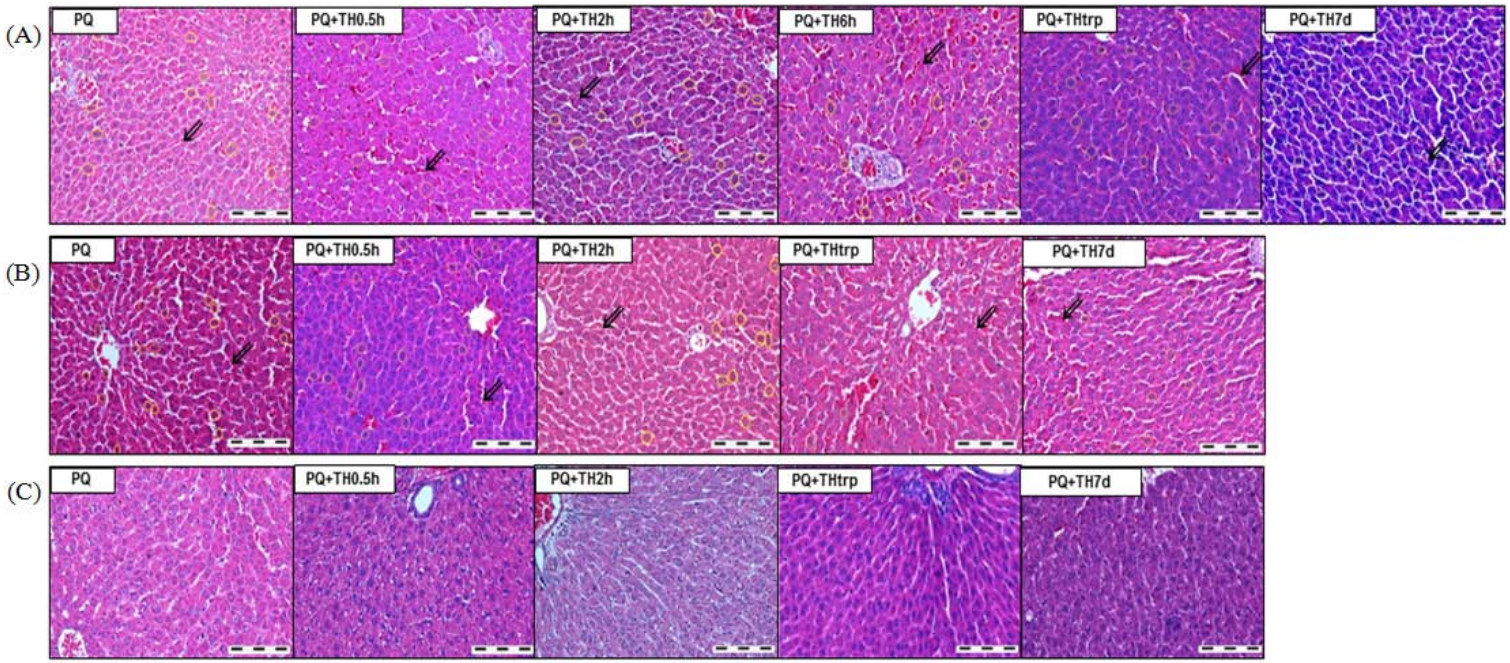

Fig. 3: Representative photomicrographs of $\mathrm{H}$ and $\mathrm{E}$ stained liver section from rats sacrificed or found dead (A) within four days ( $\leq 96 \mathrm{~h}$ ), (B)within four to seven days (96-168 h) and (C) at the end of the experimental period (day 28) following PQ intoxication (scale bar: 100 $\mu \mathrm{m}$; original magnification: $\times 200$ ). The major histological changes observed in these rats include dilated and congested liver sinusoids

(double arrow). Additionally, there was a notable increase in binucleated hepatocytes (yellow circles), one of the features of liver regeneration in response to injury.[PQ, paraquat group; TH, Tualang honey (single-dose honey treatment at 0.5 (TH0.5h), 2 (TH2h) or $6 \mathrm{~h}$ (TH6h); multiple-dose honey treatment at 0.5, 2 and $6 \mathrm{~h}$ (THtrp) or $7 \mathrm{~d}$ of consecutive treatment (TH7d)]

Overall, although treatment with single or multiple doses of $\mathrm{TH}$ at various times of administration failed to improve the survival rate in acute PQ-intoxicated rats, it may reduce or delay the toxic effects of $\mathrm{PQ}$ as evidenced by the post-mortem histological analyses in the major organs in the honey-treated groups.

\section{ACKNOWLEDGEMENT}

The authors would like to acknowledge staff from the Pharmacology Department and the Animal Research and Service Center for their help in this study. This study was partly supported by the Universiti Sains Malaysia RU Grant (1001/PPSP/813055).

\section{CONFLICT OF INTERESTS}

The authors declare that there is no conflict of interest regarding the publication of this paper.

\section{REFERENCES}

1. Eddleston M, Bateman DN. Pesticides. Medicine 2007;35:646-8.

2. Bus JS, Gibson JE. Paraquat: model for oxidant-initiated toxicity. Environ Health Perspect 1984;55:37-46.

3. Abdollahi M, Ranjbar A, Shadnia S, Nikfar S, Rezaie A. Pesticides and oxidative stress: a review. Med Sci Monit 2004;10:RA141-7.

4. Halliwell B. The wanderings of a free radical. Free Radical Biol Med 2009;46:531-42.

5. Kale MA, Bindu SM, Khadkikar P. Role of antioxidants and nutrition in oxidative stress: a review. Int J Appl Pharm 2015;7:1-4.

6. Franzen D, Baer F, Heitz W, Mecking H, Eidt S, Kaferstein H, et al. Failure of radiotherapy to resolve fatal lung damage due to paraquat poisoning. Chest 1991;100:1164-5.

7. Lin JL, Wei MC, Liu YC. Pulse therapy with cyclophosphamide and methylprednisolone in patients with moderate to severe paraquat poisoning: a preliminary report. Thorax 1996;51:661-3.

8. Gawarammana IB, Buckley NA. Medical management of paraquat ingestion. Br J Clin Pharmacol2011;72:745-57.

9. Koh KH, Tan CHH, Hii LWS, Lee J, Ngu LLS, Chai AJM, et al. Survival predictors in paraquat intoxification and role of immunosuppression. Toxicol Rep 2014;1:490-5.

10. Suntres ZE. Role of antioxidants in paraquat toxicity. Toxicology 2002;180:65-77.

11. Ilizarov AM, Koo HC, Kazzaz JA, Mantell LL, Li Y, Bhapat R, et al. Overexpression of manganese superoxide dismutase protects lung epithelial cells against oxidant injury. Am J Respir Cell Mol Biol 2001;24:436-41.
12. Muzykantov VR. Delivery of antioxidant enzyme proteins to the lung. Antioxid Redox Signal 2001;3:39-62.

13. Han J, Ma D, Zhang M, Yang X, Tan D. Natural antioxidant betanin protects rats from paraquat-induced acute lung injury interstitial pneumonia. BioMed Res Int 2015 http://dx.doi.org/10.1155/2015/608174

14. Chen Y, Nie YC, Luo YL, Lin F, Zheng YF, Cheng GH, et al. Protective effects of naringin against paraquat-induced acute lung injury and pulmonary fibrosis in mice. Food Chem Toxicol 2013;58:133-40.

15. Saba ZH, Suzana M, Yasmin AM. Honey: food or medicine. Med Health 2013;8:3-18.

16. Küçük M, KolaylI S, Karaoglu S, Ulusoy E, BaltacI C, Candan F. Biological activities and chemical composition of three honeys of different types from Anatolia. Food Chem 2007;100:526-34.

17. Nagai $T$, Inoue $R$, Kanamori $N$, Suzuki $N$, Nagashima $T$. Characterization of honey from different floral sources. Its functional properties and effects of honey species on storage of meat. Food Chem 2006;97:256-62.

18. Fauzi AN, Norazmi MN, Yaacob NS. Tualang honey induces apoptosis and disrupts the mitochondrial membrane potential of human breast and cervical cancer cell lines. Food Chem Toxicol 2011;49:871-8.

19. Ghashm AA, Othman NH, Khattak MN, Ismail NM, Saini R Antiproliferative effect of Tualang honey on oral squamous cell carcinoma and osteosarcoma cell lines. BMC Complementary Altern Med 2010;10:49.

20. Erejuwa OO, Sulaiman SA, Wahab MS, Salam SK, Salleh MS, Gurtu S. Antioxidant protective effect of glibenclamide and metformin in combination with honey in pancreas of streptozotocin-induced diabetic rats. Int J Mol Sci 2010;11:2056-66.

21. Khoo YT, Halim AS, Singh KK, Mohamad NA. Wound contraction effects and antibacterial properties of Tualang honey on fullthickness burn wounds in rats in comparison to hydrofibre. BMC Complementary Altern Med 2010;10:48.

22. Nasir NA, Halim AS, Singh KK, Dorai AA, Haneef MN Antibacterial properties of tualang honey and its effect in burn wound management: a comparative study. BMC Complementary Altern Med 2010;10:31.

23. Tan HT, Rahman RA, Gan SH, Halim AS, Hassan SA, Sulaiman SA, et al. The antibacterial properties of Malaysian tualang honey against wound and enteric microorganisms in comparison to manuka honey. BMC Complementary Altern Med 2009;9:34.

24. Zaid SS, Sulaiman SA, Sirajudeen KN, Othman NH. The effects of tualang honey on female reproductive organs, tibia bone and 
hormonal profile in ovariectomised rats--animal model for menopause. BMC Complementary Altern Med 2010;10:82.

25. Mohamed M, Sirajudeen K, Swamy M, Yaacob NS, Sulaiman SA. Studies on the antioxidant properties of tualang honey of Malaysia. Afr J Tradit Complementary Altern Med 2010;7:59-63.

26. Yao R, Cao Y, He YR, Lau WB, Zeng Z, Liang ZA. Adiponectin attenuates lung fibroblasts activation and pulmonary fibrosis induced by paraquat. PLoS One 2015;10:e0125169.

27. Acharya VK, Pawadshettar S, Arun M, Unnikrishnan B, Tantry BV. Penicillamine in interstitial lung disease: a timely remainder of an old foe. Asian J Pharm Clin Res 2016;9:1-3.
28. Duncan AW, Soto-Gutierrez A. Liver repopulation and regeneration: new approaches to old questions. Curr Opin Organ Transplant 2013;18:197-202.

29. Shalini S, Kumar S. Caspase-2 and the oxidative stress response. Mol Cell Oncol 2015;2:e1004956.Doi:10.1080/23723556.2015. 1004956

\section{How to cite this article}

- Suk Peng Tang, Hasnan Jaafar, Siew Hua Gan, Kuttulebbai NS Sirajudeen, Siti Amrah Sulaiman. Survival time and histological outcome of major organs following honey administration to acute paraquat-intoxicated rats: a preliminary study.Int J Pharm Pharm Sci 2017;9(2):319-323. 